\title{
Tum-1, a tumstatin fragment, gene delivery into hepatocellular carcinoma suppresses tumor growth through inhibiting angiogenesis
}

\author{
TAKASHI GOTO ${ }^{1}$, HIROKI ISHIKAWA ${ }^{1}$, KOJIRO MATSUMOTO ${ }^{1}$, DAISUKE NISHIMURA ${ }^{1}$, \\ MARIKO KUSABA $^{1}$, NAOTA TAURA ${ }^{1}$, HIDETAKA SHIBATA ${ }^{1}$, HISAMITSU MIYAAKI ${ }^{1}$ \\ TATSUKI ICHIKAWA ${ }^{1}$, KEISUKE HAMASAKI ${ }^{1}$, KAZUHIKO NAKAO ${ }^{1}$, \\ YOHEI MAESHIMA ${ }^{2}$ and KATSUMI EGUCHI ${ }^{1}$
}

\author{
${ }^{1}$ First Department of Internal Medicine, Nagasaki University School of Medicine, 1-7-1 Sakamoto, Nagasaki 852-8501; \\ ${ }^{2}$ Department of Medicine and Clinical Science, Okayama University Graduate School of Medicine, \\ Dentistry and Pharmaceutical Sciences, Okayama, Japan
}

Received December 27, 2007; Accepted February 5, 2008

\begin{abstract}
Since hepatocellular carcinoma (HCC) is a hypervascular cancer, anti-angiogenic therapy is a promising approach to treat HCC. In the present study, we investigated the antiangiogenic and antitumor effects of tum-1, a fragment of tumstatin, gene transduction into HCC in vitro and in vivo. Tum-1 gene was cloned into a pSecTag2B mammalian expression vehicle to construct pSecTag2B-tum-1. pSecTag2B-tum-1 or vehicle were transfected into human HCC cells, PLC/PRF/5 cells stably and Huh-7 cells transiently. pSecTag2B-tum-1 transfection slightly repressed the proliferation of both PLC/PRF/5 and Huh-7 cells in vitro. Addition of conditioned media (CM) from tum-1 expressing $\mathrm{PLC} / \mathrm{PRF} / 5$ cells significantly inhibited the spontaneous and vascular endothelial growth factor (VEGF)-induced proliferation and migration of human umbilical vein endothelial cells (HUVEC) in vitro with diminishing the VEGF-induced phosphorylation of both Akt and extracellular signal-regulated kinase (ERK) that are known to mediate VEGF-induced proliferation and migration of endothelial cells. In in vivo experiments, intratumoral injection of pSecTag2B-tum-1 significantly repressed the growth of pre-established Huh-7 tumors in athymic mouse models accompanying the decreased density of CD34 positive vessels in tumors. In conclusion, our results suggest that antiangiogenic gene therapy using tum-1 gene may be an efficient strategy for the treatment of HCC.
\end{abstract}

Correspondence to: Dr Kazuhiko Nakao, First Department of Internal Medicine, Nagasaki University School of Medicine, 1-7-1 Sakamoto, Nagasaki 852-8501, Japan

E-mail: kazuhiko@net.nagasaki-u.ac.jp

Key words: tum-1, tumstatin, hepatocellular carcinoma, angiogenesis

\section{Introduction}

Hepatocellular carcinoma (HCC) is one of the most common fatal malignancies worldwide, and is especially common in several parts of Asia and Africa (1). Although advances in medical technology have permitted the early recognition and treatment of HCC $(1,2)$, the annual death rate from HCC exceeds 30,000 in Japan (3). Therefore, there is a need to develop new strategies to treat HCC.

Recently, it has been reported that antiangiogenic agents sufficiently inhibit tumor growth in vivo (4). Since HCC is a hypervascular cancer, antiangiogenic therapy might be particularly effective in the treatment of patients with HCC. However, systemic administration of antiangiogenic agents, such as thalidomide (5) or TNP-470 (6), might not be the most efficient method for locally aggressive tumors. Therefore, it is conceivable that antiangiogenic gene delivery to cancer cells could be suitable for the treatment of HCC, where it can increase the local concentration of therapeutic endogenous agents. In fact, we have reported that the introduction of antiangiogenic genes such as angiostatin and pigment epithelium-derived factor (PEDF) genes into HCC cells exhibited significant antitumor effect in xenograft models $(7,8)$.

The noncollagenous domain of $\alpha 3$ chain of type IV collagen, namely tumstatin, has the antiangiogenic property by inhibiting endothelial cell proliferation and inducing their apoptosis via an interaction with avß3 integrin (9-15). Antiangiogenic effect of tumstatin has been studied in xenograft models, where tumstatin repressed the growth of several cancer types including renal cell carcinoma, prostate cancer, melanoma and lung carcinoma $(9-11,13)$. In the present study, we constructed a mammalian expression vector expressing tum-1 which consists of 54-244 amino acids of tumstatin and has antiangiogenic activity (9). Following stable transfection of this vector into HCC cells, we examined the antiangiogenic activity of tum-1 using cultured human umbilical vein endothelial cells (HUVEC) in the presence or 
absence of vascular endothelial growth factor (VEGF). In addition, we investigated the antiangiogenic and antitumor effects of tum-1 in vivo by intratumoral injection of tum-1 expression vector into HCC tumor implanted subcutaneously in athymic nude mice.

\section{Materials and methods}

Cell culture. Human HCC cell lines, PLC/PRF/5 and Huh-7 cells, were maintained in RPMI supplemented with $10 \%$ bovine calf serum. Human umbilical vascular endothelial cells (HUVEC) were purchased from Sankyo Junyaku (Tokyo, Japan) and were grown in endothelial cell growth medium 2. HUVEC were grown to $<6$ passages for all experiments.

Plasmid construction and transfection. The human tum-1 cDNA was kindly provided by Dr Y. Maeshima (Department of Medicine and Clinical Science, Okayama University, Japan) and cloned into pSecTag2B mammalian expression vector containing hexahistidine tag (Invitrogen, Carlsbad, CA) to construct pSecTag2B-tum-1. To establish the PLC/PRF/5 cells stably expressing tum-1/hexahistidine chimera protein, $10 \mu \mathrm{g}$ of pSecTag2B-tum-1 was transfected into the cells by the lipofection method (Life Technologies, Inc., Gaithersburg, MD). After transfection, the cells were cultured in fresh medium containing Zeocin $(100 \mu \mathrm{g} / \mathrm{ml})$ for 2 weeks. Zeocinresistant pooled populations were subjected to further studies. As a control, pSecTag2B vehicle was also stably transfected into $\mathrm{PLC} / \mathrm{PRF} / 5$ cells. In addition, $\mathrm{pSecTag} 2 \mathrm{~B}$ vehicle or pSecTag2B-tum-1 was transiently transfected into Huh-7 cells.

Preparation of conditioned media. PLC/PRF/5 cells stably transfected with pSecTag2B-tum-1 or vehicle were plated on 100-mm dishes. After $24 \mathrm{~h}$, the medium was replaced with $5 \mathrm{ml}$ serum-free RPMI and incubated for $48 \mathrm{~h}$. Then, conditioned media (CM) from pSecTag2B-tum-1-transfected PLC/PRF/5 cells (CM-tum-1), vehicle-transfected PLC/PRF/5 cells (CM-Mock) and non-transfected PLC/PRF/5 cells (CM-N) were collected and used in the assay. In some experiments, CM-tum-1 was passed through the His trap kit (Amersham Life Science, Buckinghamshire, UK) and the flow-through fraction (CM-tum-1 $\Delta$ His) was collected and used in the assay. Similarly, CM from Huh-7 cells transiently transfected with SecTag2B-tum-1 (CM-tum-1) and vehicle (CM-Mock) or CM from non-transfected Huh-7 cells (CM-N) were collected and used in the assay.

Proliferation of hepatoma cells. PLC/PRF/5 cells stably transfected with vehicle (Mock) or pSecTag2B-tum-1 (tum-1) were seeded onto 96 -well culture plates at $\sim 5 \times 10^{3}$ cells/well and were incubated for $72 \mathrm{~h}$. Cell proliferation was evaluated using the CellTiter 96 Aqueous One Solution Cell Proliferation Assay kit (Promega, Madison, WI). Similarly, non-transfected PLC/PRF/5 cells were seeded onto 96-well culture plates. After $24 \mathrm{~h}$, the medium was replaced with $100 \mu \mathrm{l}$ of CM-N, CM-Mock, CM-tum-1 and CM-tum- $1 \Delta$ His. After $48 \mathrm{~h}$, cell proliferation was determined. Whereas, Huh-7 cells seeded onto 96-well culture plates were transiently transfected with vehicle (Mock) or pSecTag2B-tum-1 (tum-1) and cultured for $48 \mathrm{~h}$, and cell proliferation was determined.
Proliferation and migration of HUVEC. HUVECs were plated onto 96-well culture plates at approximately $5 \times 10^{3}$ cells/well and incubated for $24 \mathrm{~h}$. Medium was replaced with $100 \mu \mathrm{l}$ of CM-Mock or CM-tum-1 with or without $10 \mathrm{ng} / \mathrm{ml}$ of recombinant human vascular endothelial growth factor (VEGF, R\&D systems, Minneapolis, MN, USA). After 48 h, cell proliferation was determined by the CellTiter 96 Aqueous One Solution Cell Proliferation Assay kit. Migration of HUVEC was analyzed using 8.0- $\mu \mathrm{m}$ 24-well Transwells (Corning, Acton, MA) as described previously $(7,8)$. Briefly, $600 \mu \mathrm{l}$ of CM-Mock or CM-tum-1 with or without $10 \mathrm{ng} / \mathrm{ml}$ of VEGF was placed in the lower chamber. HUVEC $\left(\sim 2 \times 10^{4}\right)$ suspended in $200 \mu 1$ of serum-free RPMI were added to the upper chamber. After 24-h incubation, nonmigrating cells were removed from the upper surface of the membrane with a cotton swab. Cells migrating to the lower surface were fixed with methanol and stained with Giemsa. Cell number was counted with a light microscope under a high power field (magnification x200).

Western blotting. HUVEC were incubated with CM-Mock or CM-tum-1 in the presence or absence of $10 \mathrm{ng} / \mathrm{ml}$ of VEGF for $30 \mathrm{~min}$. Then, the cells were washed twice with phosphatebuffered saline (PBS), lysed by addition of lysis buffer [50 mM Tris ( $\mathrm{pH} 8.0), 150 \mathrm{mM} \mathrm{NaCl}, 0.02 \%$ sodium azide, $0.1 \%$ SDS, $100 \mu \mathrm{g} / \mathrm{ml} \mathrm{PMSF}, 1 \mu \mathrm{g} / \mathrm{ml}$ of aprotinin, $1 \% \mathrm{NP} 40$, $0.5 \%$ sodium deoxycholate and $1 \mathrm{mM}$ sodium o-vanadate] for $10 \mathrm{~min}$ at $4^{\circ} \mathrm{C}$, and insoluble materials were removed by centrifugation at $14,000 \mathrm{rpm}$ for $30 \mathrm{~min}$ at $4^{\circ} \mathrm{C}$. The supernatant was collected, and the protein concentration was determined using a Bio-Rad protein assay kit (Melville, NY, USA). The same amount of protein from each lysate or conditioned medium (10 $\mu \mathrm{g} / \mathrm{well})$ was analyzed by electrophoresis on 8-12\% SDS polyacrylamide gel and transblotted onto nitrocellulose membrane. Blots were blocked with a solution of $5 \%$ non-fat dry milk/Tris-buffered saline containing $0.1 \%$ Tween-20 (TBS-T) for $1 \mathrm{~h}$ and then incubated overnight at $4^{\circ} \mathrm{C}$ in the presence of rabbit anti-hexahistidine (ICN, Costa Mesa, CA), rabbit anti-human phosphor-Akt, rabbit antihuman Akt, rabbit anti-human phosphor-ERK1/2 and rabbit anti-human ERK1/2 (Cell Signaling, Beverly, MA, USA). The membranes were washed with TBS-T and were incubated with horseradish peroxidase-conjugated anti-rabbit immunoglobulin G. After washing with TBS-T, immunoreactive bands were visualized using the ECL chemiluminescence system (Amersham).

In vivo study. Four-week-old male BALB/c nu/nu athymic mice were purchased from Charles River (Yokohama, Japan). Animal experiments were performed in accordance with institutional guidelines, and the study was approved by the Ethics Committee of Nagasaki University. Huh-7 cells $\left(3 \times 10^{6}\right)$ were implanted subcutaneously into the left thigh. Tumor volume was calculated as follows; tumor volume = length $(\mathrm{mm}) \times$ width $^{2}(\mathrm{~mm}) \times 1 / 2$. When the tumor volume reached $100 \mathrm{~mm}^{3}$, pSecTag2B-tum-1 [75 $\mu \mathrm{g}$ plasmid/100 $\mu \mathrm{l}$ of TE (Tris ethylenediamine tetra acetic acid) buffer] with $20 \mu 1$ of lipofectin was injected into the tumor at day 1, 8, 15 and 22 . As a control, pSecTag2B vehicle (75 $\mu \mathrm{g}$ plasmid/100 $\mu 1$ of TE) with lipofectin was injected similarly. Each group consisted 


\section{A}

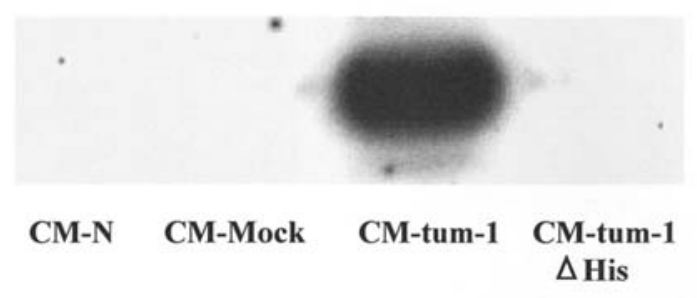

B

C
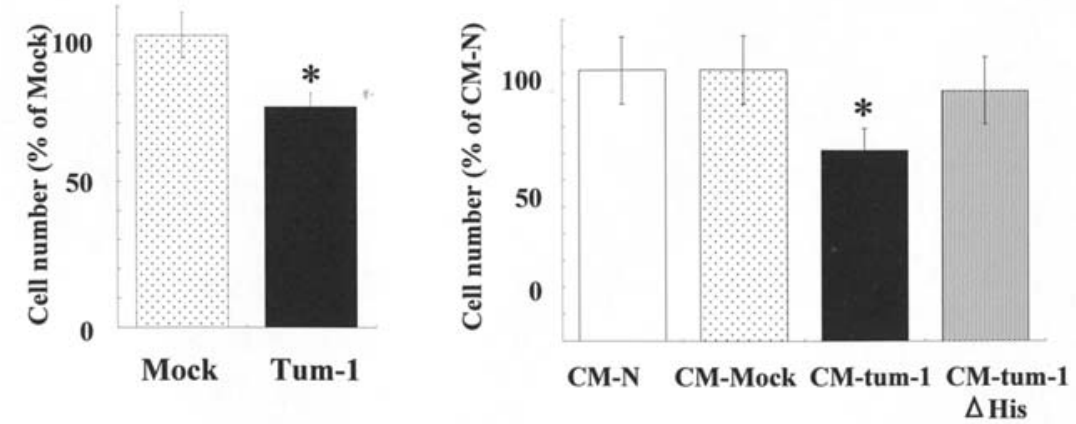

Figure 1. Growth of PLC/PRF/5 cells stably transfected with pSecTag2B-tum-1. (A) Expression of tum-1/hexahistidine chimera protein in CM from PLC/PRF/5 cells stably transfected with pSecTag2B-tum-1 was analyzed by Western blotting; CM-N, CM from non-transfected cells; CM-Mock, CM from vehicle transfected cells; CM-tum-1, CM from pSecTag2B-tum-1 transfected cells; CM-tum-1 $\Delta$ His, CM-tum-1 passed through His trap kit. (B) PLC/PRF/5 cells stably transfected with vehicle (Mock) or pSecTag2B-tum-1 (Tum-1) were cultured for $72 \mathrm{~h}$, after which cell number was counted. Results are expressed as a percentage of Mock. Data represent the mean \pm SD values of four separate experiments; " $\mathrm{p}<0.01$ vs Mock. (C) CM shown in (A) was added to nontransfected PLC/PRF/5 cells, and cells were cultured for $48 \mathrm{~h}$, then cell number was counted. Results are expressed as a percentage of CM-N. Data represent the mean \pm SD values of four separate experiments; ${ }^{*} \mathrm{p}<0.01$ vs CM-N, CM-Mock and CM-tum- $1 \Delta$ His.

of five mice. Tumor volume was measured every 2-3 days until 25 days after first injection. Tumors were removed and analyzed by reverse-transcriptase polymerase chain reaction (RT-PCR) and immunohistochemistry.

$R T-P C R$. Total RNA was extracted from tumors and PLC/ $\mathrm{PRF} / 5$ cells stably transfected pSecTag2B-tum-1. RNA was used after contaminating DNA was completely removed by DNase I treatment. RT-PCR was performed according to the instructions provided by the supplier of the OneStep RT-PCR Kit (Qiagen, Valencia, CA), using primers specific for tum-1 derived from pSecTag2B-tum-1 and glyceraldehyde 3phosphate dehydrogenase (GAPDH) as a control. Reaction mixtures $(10 \mu 1)$ were loaded on $1.2 \%$ agarose gels and visualized by ethidium bromide staining.

Immunohistochemistry. Immunohistochemistry was performed using anti-mouse CD34 antibody (Serotec Ltd., Oxford, UK ) and anti-mouse $\alpha$ SMA (Actin, Smooth Muscle, Shandon Immunon $^{\mathrm{TM}}$, USA). Tissue samples of the tumor extracted from each mouse were cut into $4-\mu \mathrm{m}$-thick sections and mounted on aminopropyltriethoxysilane-coated glass slides. Sections were immunostained with anti-CD34 at a dilution of
1:100 for 60 min using the Streptavidin Peroxidase technique (SAB) (Histomouse ${ }^{\mathrm{TM}}$ Plus Kits, Zymed Labolatories Inc., South San Francisco, USA). The second staining with antiaSMA was conduced by making the section reactive primary antibody, anti- $\alpha \mathrm{SMA}$ (dilution $1: 100$ ), at $4^{\circ} \mathrm{C}$ overnight, and by using the SAB technique. The sections were stained with the mixture of a commercial chromogen (VIP) and hydrogen peroxide and hematoxylin for counterstaining.

Statistical analysis. All data were expressed as mean \pm SD. Differences between groups were examined for statistical significance using Student's t-test. All reported p-values are two-tailed, and those $<0.05$ were considered statistically significant.

\section{Results}

Tum-1 gene introduction slightly represses the proliferation of HCC cells. Tum-1 expression plasmid (pSecTag2B-tum-1) was stably transfected into PLC/PRF/5 cells, and the secretion of tum-1 protein from the cells was analyzed by Western blotting. As shown in Fig. 1A, conditioned medium (CM) from PLC/PRF/5 cells stably transfected with pSecTag2B- 
A
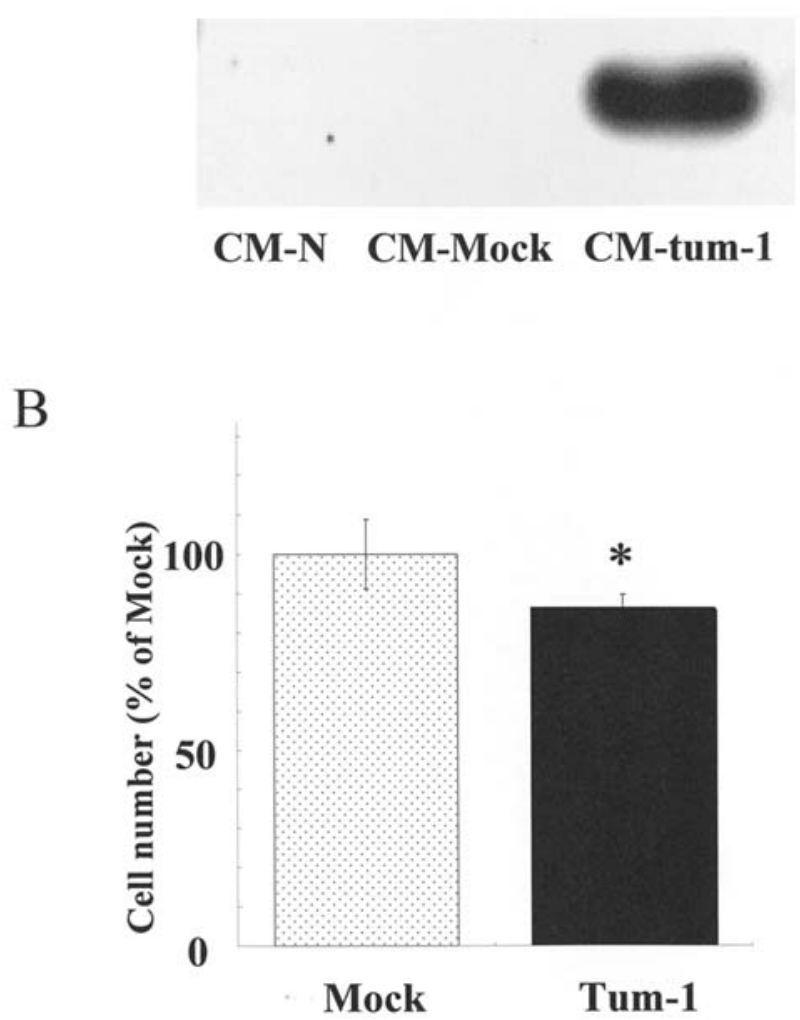

Figure 2. Growth of Huh-7 cells transiently transfected with pSecTag2Btum-1. (A) Expression of tum-1/hexahistidine chimera protein in CM from Huh-7 cells transiently transfected with pSecTag2B-tum-1 was analyzed by Western blotting with using anti-His tag antibody, CM-N, CM from nontransfected cells; CM-Mock, CM from vehicle transfected cells; CM-tum-1, CM from pSecTag2B-tum-1 transfected cells. (B) Huh-7 cells transiently transfected with pSecTag2B-tum-1 (Tum-1) or vehicle (Mock) were incubated for $48 \mathrm{~h}$, after which cell number was counted. Results are expressed as a percentage of Mock. Data represent the mean \pm SD values of four separate experiments; ${ }^{\mathrm{p}}<0.05$ vs Mock.

tum-1 (CM-tum-1) contained the tum-1/hexahistidine chimera protein detected by anti-hexahistidine antibody, and this chimera protein was effectively removed by the His trap kit (CM-tum-1 $\Delta$ His) (Fig. 1A). Cell proliferation assay showed that the growth of PLC/PRF/5 cells stably transfected with pSecTag2B-tum-1 was slightly slower than that of PLC/PRF/5 cells stably transfected with vehicle (Mock) (Fig. 1B). To elucidate whether this growth suppression was mediated by tum-1/hexahistidine chimera protein, CM from PLC/PRF/5 cells stably transfected with pSecTag2B-tum-1 (CM-tum-1) was added to parental PLC/PRF/5 cells, which also retarded the growth of parental cells compared with addition of CMtum-1 passed through His trap kit (CM-tum-1 $\Delta$ His) or $\mathrm{CM}$ from PLC/PRF/5 cells stably transfected with vehicle (CMMock) (Fig. 1C). Next, pSecTag2B-tum-1 was transiently transfected into Huh-7 cells, and effect on the cell growth was determined. As shown in Fig. 2A, CM from Huh-7 cells transiently transfected with pSecTag2B-tum-1 (CM-tum-1) contained the tum-1/hexahistidine chimera protein. The growth of Huh-7 was slightly repressed by transient transfection with pSecTag2B-tum-1 (Fig. 2B). These results suggest that tum-1 has a weak but significant antiproliferative activity against human HCC cells.

Tum-1 inhibits the spontaneous and VEGF-induced proliferation and migration of HUVEC. Next, we determined the effects of tum-1 on the spontaneous and VEGF-induced proliferation and migration of HUVEC. As shown in Fig. 3A, addition of $\mathrm{CM}$ from $\mathrm{PLC} / \mathrm{PRF} / 5$ cells stably transfected with pSecTag2B-tum-1 (CM-tum-1) clearly inhibited both spontaneous and VEGF-induced proliferation of HUVEC compared with addition of CM from vehicle transfected cells (CM-Mock) (Fig. 3A). Similarly, addition of CM-tum-1 repressed the spontaneous and VEGF-induced migration of HUVEC (Fig. 3B). Since phosphatidylinositol 3-kinase (PI3K)-protein kinase B (PKB/Akt) and extracellular signalregulated kinase (ERK) signaling pathways are involved in the VEGF-induced proliferation and migration of HUVEC (16-18), we analyzed the effects of tum-1 on the VEGF-induced phosphorylation of Akt and ERK by Western blotting (Fig. 4). Phosphorylation of both Akt and ERK was constitutively detected in HUVEC, which was further upregulated by VEGF. However, addition of CM-tum-1 repressed the VEGF-induced phosphorylation of both Akt and ERK to the basal level.

Antitumor effect of tum-1 gene introduction in vivo. Huh-7 cells were subcutaneously implanted and tumors were established in athymic nude mice because Huh-7 cells were more efficiently transplantable than other cell lines. After reaching an adequate size, the tumor was directly injected with pSecTag2B-tum-1, and the effect of treatment on tumor size was determined. Injection of pSecTag2B-tum-1 resulted in a significant reduction of tumor volume compared with vehicle-injected tumors at day $24(\mathrm{p}<0.01$; Fig. 5). The expression of tum-1 mRNA in the pSecTag2B-tum-1-injected tumors (at day 24) was confirmed by RT-PCR (Fig. 6A), where the density of CD34 and $\alpha$ SMA positive vessels/fields was significantly lower than those in the vehicle-injected tumors (Fig. 6B and C). These results indicate that the tumor suppressive effect of tum-1 gene injection was closely related to the vascularity of tumors.

\section{Discussion}

Tumstatin, a non-collagenous domain of $\alpha 3$ chain of type IV collagen, consists of 244 amino acids $(9,14)$, which is cleaved from type IV collagen by matrix metalloproteinase-9 $(14,19)$. Tumstatin inhibits proliferation and causes apoptosis of endothelial cells through avß3 integrin interaction in an RGD-independent manner (9-14). Tumstatin also inhibits tube formation of endothelial cells on Matrigel and induces G1 endothelial cell cycle arrest (14). Deletion mutants of tumstatin including tum-1 (54-244 amino acids), tum-2 (1-132 amino acids) and tum-5 (54-132 amino acids) retain the antiangiogenic activity (9-11).

In the present study, tum-1 expression plasmid vector (pSecTag2B-tum-1) was introduced into HCC cells. HCC cells transfected with pSecTag2B-tum-1 produced the detectable tum-1/hexahistidine chimera protein in its CM. Surprisingly, stable and transient tum-1 gene transfection into PLC/PRF/5 and Huh-7 cells, respectively, slightly repressed 
A HUVEC Proliferation assay



\section{B HUVEC Migration assay}
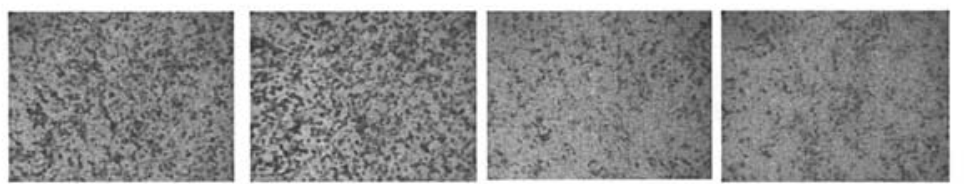

CM-Mock

CM-Mock + VEGF

CM-tum-1

CM-tum-1 + VEGF

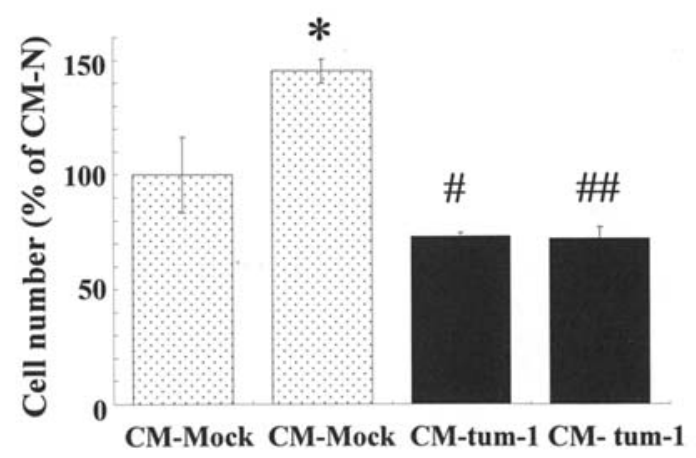

VEGF

$(10 \mathrm{ng} / \mathrm{ml})$

Figure 3. Effects of CM containing tum-1/hexahistidine chimera protein on proliferation and migration of HUVEC. (A) HUVEC were cultured on 96-well plates in $100 \mu 1$ of CM derived from PLC/PRF/5 cells transfected with vehicle (CM-Mock) or pSecTag2B-tum-1 (CM-tum-1) in the presence or absence of $\operatorname{VEGF}(10 \mathrm{ng} / \mathrm{ml})$. Cell number was determined after 48-h incubation. Results are expressed as a percentage of CM-Mock without VEGF. Data represent the mean \pm SD values of four separate experiments; ${ }^{*} \mathrm{p}<0.01$ vs CM-Mock without VEGF; ${ }^{*} \mathrm{p}<0.05$ vs CM-Mock without VEGF; ${ }^{\# / p} \mathrm{p}<0.01$ vs CM-Mock with VEGF. (B) CM-Mock or CM-tum-1 with or without VEGF was placed in the lower chamber of a modified Boyden chamber, and HUVEC were seeded to the upper chamber. After 24-h incubation, non-migrating cells were removed and cells that migrated through the membrane pores were stained with Giemsa and counted. Representative micrographs of migrated HUVEC in each CM are shown in upper panel. Results are expressed as a percentage of CM-Mock without VEGF. Data represent the mean \pm SD values of four separate experiments; " $\mathrm{p}<0.01$ vs CM-Mock without VEGF; ${ }^{*} \mathrm{p}<0.05$ vs CM-Mock without VEGF; ${ }^{\# \#} \mathrm{p}<0.01$ vs CM-Mock with VEGF.

the proliferation of these cells. Recent reports revealed that $\alpha v B 3$ integrin, the receptor of tumstatin, is expressed in several HCC cell lines including Huh-7 cells (20) and also in clinical samples of HCC (21). Therefore, it is possible that tum-1 could inhibit the proliferation of HCC cells through interacting with $\alpha v \beta 3$ integrin. Similar observation was reported that the gene introduction of tumstatin or its Cterminal residues $185-203$ into melanoma cells directly inhibited the proliferation and invasiveness of these cells in vitro (22).
A potential mechanism of antiangiogenic function of tumstatin has been reported that tumstatin inhibits activation of focal adhesion kinase (FAK), phosphatidylinositol 3kinase (PI3K), protein kinase B (PKB/Akt), and mammalian target of rapamycin (mTOR), and it prevents the dissociation of eukaryotic initiation factor 4E protein (eIF4E) from 4E-binding protein 1 , resulted in the inhibition of cap-dependent protein synthesis in endothelial cells $(23,24)$. Since PI3K-PKB/Akt signaling plays a key role in cell growth and survival in a variety of cells (25), it is conceivable that inhibition of this 
A

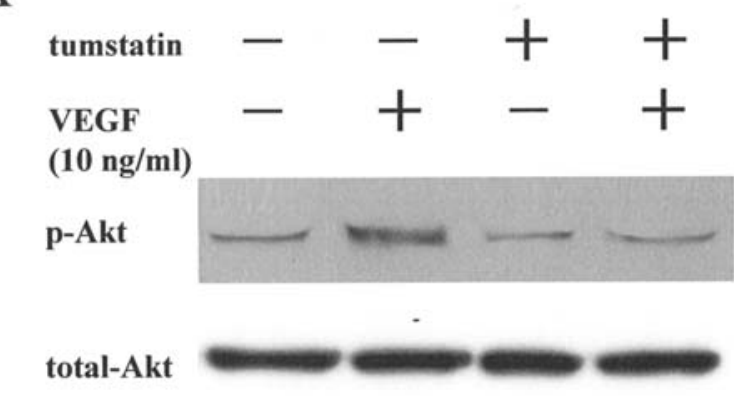

CM-Mock CM-Mock CM-tum-1 CM- tum-1

B



CM-Mock CM-Mock CM-tum-1 CM- tum-1

Figure 4. Effects of CM containing tum-1/hexahistidine chimera protein on spontaneous or VEGF-induced phosphorylation of Akt and ERK. Huh-7 cells were incubated with indicated CM with or without $10 \mathrm{ng} / \mathrm{ml}$ of VEGF for $30 \mathrm{~min}$, and the levels of phosphorylation of Akt (A) or ERK (B) were analyzed by Western blotting. Results shown are from one representative experiment from a total of four performed.

signaling leads to the growth arrest and apoptosis of endothelial cells. Furthermore, recent studies revealed that VEGF promoted angiogenesis through activating PI3K-PKB/Akt signaling $(16,17)$, and that luteolin, an antiangiogenic compound, repressed the VEGF-induced angiogenesis by inhibiting PI3K-PKB/Akt signaling (26). In contrast to tumstatin, endostatin, another antiangiogenic fragment released from $\alpha 1$ chain of type XVIII collagen, interacts with $\alpha 5 \beta 1$ integrin and inhibits activation of FAK, and ras-raf-ERK signaling, but not PI3K-PKB/Akt signaling, and it prevents endothelial cell migration with no effect on proliferation and survival (24), indicating that tumstatin has a distinct antiangiogenic mechanism from endostatin. In this study, addition of CM containing tum-1 repressed not only spontaneous and VEGFinduced proliferation of HUVEC but also its migration, and clearly abolished the VEGF-induced phosphorylation of both Akt and ERK in HUVEC. This is surprising because tumstatin has been reported to inhibit, through binding to $\alpha v B 3$, the PI3K-PKB/Akt signaling and proliferation of endothelial cells rather than ras-raf-ERK signaling and migration of
A

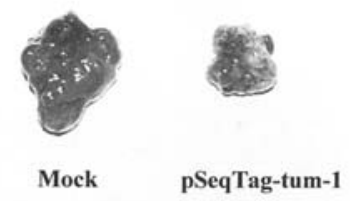

B

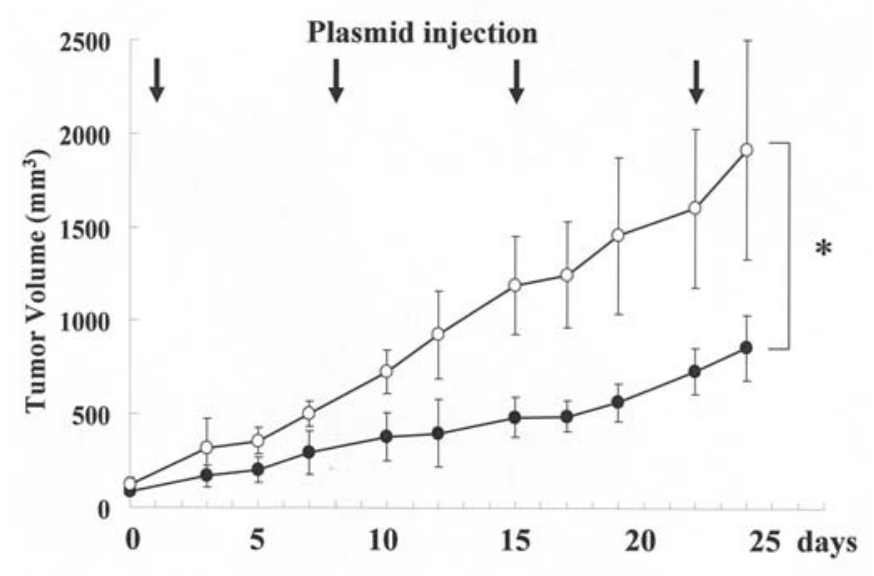

Figure 5. Inhibition of pre-established Huh-7 tumor growth by injection of pSecTag2B-tum-1 in athymic mice. Mock (vehicle; open circle) or pSecTag2B-tum-1 (closed circle) was injected intratumorally into preestablished tumors of Huh-7 cells. Mice were sacrificed on day 25, and subcutaneous tumors were extracted. (A) Representative photographs of harvested tumors. (B) Serial changes in tumor volume in the two groups. Data are mean $\pm \mathrm{SD}(\mathrm{n}=5) ;{ }^{*} \mathrm{p}<0.01$ vs vehicle.

these cells (24). However, several studies have reported that avß3 blocking antibody which has a similar antiangiogenic activity to tumsatain (27) inhibited the angiogenesis through blocking the ras-raf-ERK signaling (13), and that blocking of ras-raf-ERK signaling by PD98059 resulted in the inhibition of VEGF-induced proliferation of HUVEC (18). Taken together, it may be possible that there are several mechanisms mediating antiangiogenic function of tumstatin.

Non-viral gene delivery systems are less efficient at inducing transgene expression and have shorter-term expression compared with viral delivery systems. Despite the expected low efficiency of gene induction, intratumoral injection of tum-1 expression plasmid vector (pSecTag2Btum-1) significantly repressed the Huh-7 tumor growth accompanying the decreased density of CD34 and $\alpha \mathrm{SMA}$ positive vessels compared with vehicle injection. These results suggest that a sufficient bystander effect was achieved by this strategy, and if the transgene is expressed intratumorally, highly efficient therapeutic gene induction may not be necessarily required. We have reported that PEDF gene transduction into Huh-7 tumor significantly repressed its growth in athymic mouse models as well as tum-1 shown in this study, but PEDF did not directly inhibit the proliferation of Huh-7 cells in vitro (8). Therefore, it is likely that antitumor effect of tum-1 in vivo could be attributable to its anti- 


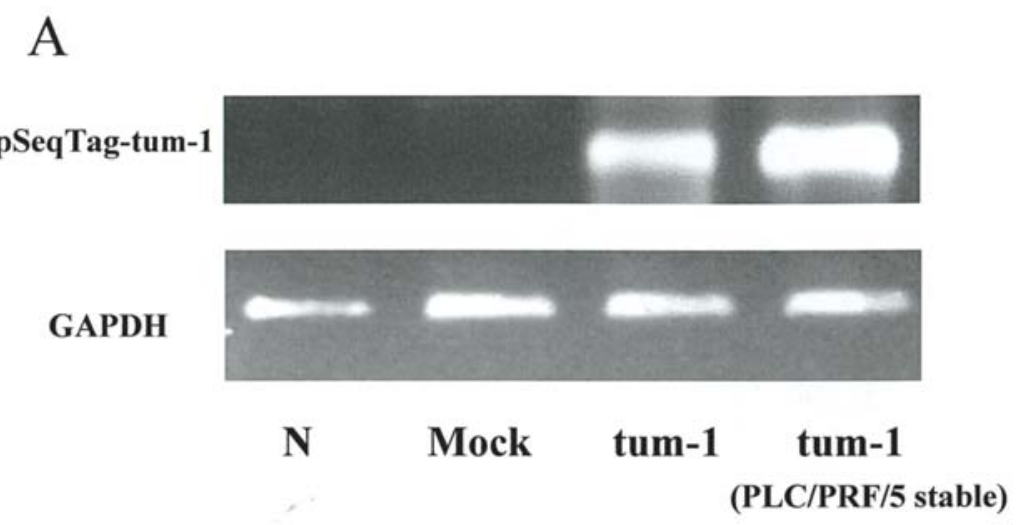

B

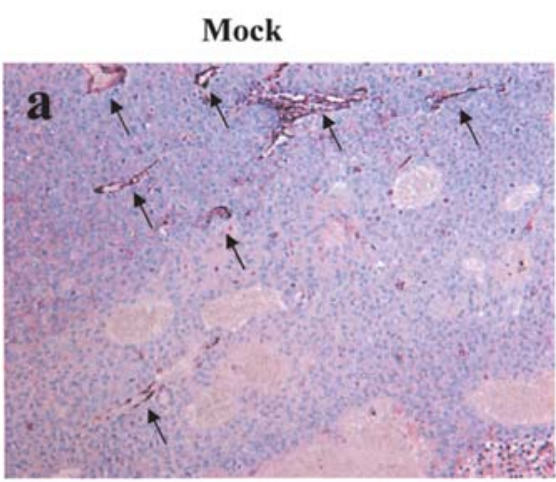

pSeqTag-tum-1

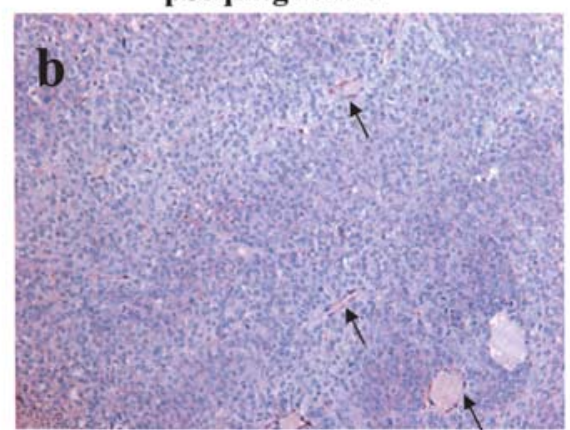

C

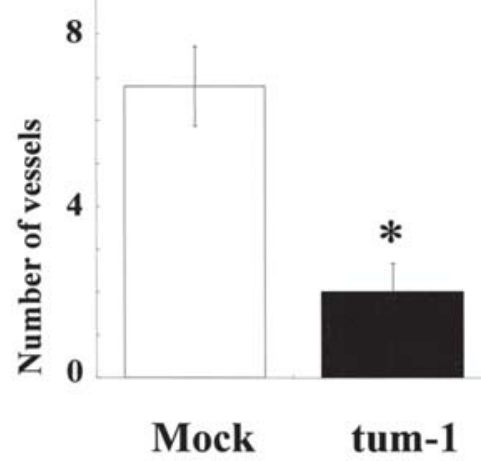

Figure 6. Tum-1 mRNA expression and CD34-positive vessel density in the tumors. (A) Exogenous tum-1 mRNA expression in the tumor was analyzed by RT-PCR. Total RNA was extracted from tumor tissues. RT-PCR was performed with primers specific for tum-1 and GAPDH (internal control). Total RNA from PLC/PRF/5 cells stably transfected with pSecTag2B-tum-1was used as a positive control (right column); N, negative control; Mock, vehicle injected tumor; tum-1, pSecTag2B-tum-1 injected tumor. (B) Representative photographs of a harvested tumor stained immunohistochemically with anti-CD34 and anti- $\alpha$ SMA antibody. Arrows indicate CD34 (brown) and $\alpha$ SMA (violet) positive vessels (original magnification x200); a, Mock (vehicle) injected tumor; b, pSecTag2B-tum-1 injected tumor. (C) Number of CD34 (brown) and $\alpha$ SMA (violet) positive vessels/field. Data represent a number of CD34 and $\alpha$ SMA positive vessels counted from the five different fields of each sample. Data are mean $\pm \operatorname{SD}(n=5) ;{ }^{*} p<0.01$ vs Mock.

angiogenic activity rather than direct antiproliferative activity against Huh-7 cells. In conclusion, it is possible that intratumoral gene injection of tumstatin or its active derivatives including tum-1 is a promising strategy for the treatment of HCC.

\section{References}

1. Llovet JM, Burroughs A and Bruix J: Hepatocellular carcinoma. Lancet 362: 1907-1917, 2003.

2. Sato Y, Nakata K, Kato Y, et al: Early recognition of hepatocellular carcinoma based on altered profiles of alpha-fetoprotein. N Engl J Med 328: 1802-1806, 1993.
3. Kiyosawa K and Tanaka E: Characteristics of hepatocellular carcinoma in Japan. Oncology 62 (Suppl. 1): 5-7, 2002.

4. Gaya AM and Rustin GJ: Vascular disrupting agents: a new class of drug in cancer therapy. Clin Oncol (R Coll Radiol) 17: 277-290, 2005.

5. Patt YZ, Hassan MM, Lozano RD, Ellis LM, Peterson JA and Waugh KA: Durable clinical response of refractory hepatocellular carcinoma to orally administered thalidomide. Am J Clin Oncol 23: 319-321, 2000.

6. Kin M, Torimura T, Ueno $\mathrm{T}$, et al: Angiogenesis inhibitor TNP-470 suppresses the progression of experimentally-induced hepatocellular carcinoma in rats. Int J Oncol 16: 375-382, 2000.

7. Ishikawa H, Nakao K, Matsumoto K, et al: Antiangiogenic gene therapy for hepatocellular carcinoma using angiostatin gene. Hepatology 37: 696-704, 2003. 
8. Matsumoto K, Ishikawa H, Nishimura D, Hamasaki K, Nakao K and Eguchi K: Antiangiogenic property of pigment epitheliumderived factor in hepatocellular carcinoma. Hepatology 40: 252-259, 2004.

9. Maeshima Y, Colorado PC, Torre A, et al: Distinct antitumor properties of a type IV collagen domain derived from basement membrane. J Biol Chem 275: 21340-21348, 2000.

10. Maeshima Y, Yerramalla UL, Dhanabal M, et al: Extracellular matrix-derived peptide binds to alpha(v)beta(3) integrin and inhibits angiogenesis. J Biol Chem 276: 31959-31968, 2001.

11. Maeshima Y, Manfredi M, Reimer C, et al: Identification of the anti-angiogenic site within vascular basement membranederived tumstatin. J Biol Chem 276: 15240-15248, 2001.

12. Bergers $\mathrm{G}$ and Benjamin LE: Tumorigenesis and the angiogenic switch. Nat Rev Cancer 3: 401-410, 2003.

13. Hutchings H, Ortega N and Plouet J: Extracellular matrix-bound vascular endothelial growth factor promotes endothelial cell adhesion, migration, and survival through integrin ligation. FASEB J 17: 1520-1522, 2003.

14. Hamano Y and Kalluri R: Tumstatin, the $\mathrm{NC1}$ domain of alpha3 chain of type IV collagen, is an endogenous inhibitor of pathological angiogenesis and suppresses tumor growth. Biochem Biophys Res Commun 333: 292-298, 2005.

15. Sund M, Hamano Y, Sugimoto H, et al: Function of endogenous inhibitors of angiogenesis as endothelium-specific tumor suppressors. Proc Natl Acad Sci USA 102: 2934-2939, 2005.

16. Cai J, Ahmad S, Jiang WG, et al: Activation of vascular endothelial growth factor receptor-1 sustains angiogenesis and Bcl-2 expression via the phosphatidylinositol 3-kinase pathway in endothelial cells. Diabetes 52: 2959-2968, 2003.

17. Abid MR, Guo S, Minami T, et al: Vascular endothelial growth factor activates PI3K/Akt/forkhead signaling in endothelial cells. Arterioscler Thromb Vasc Biol 24: 294-300, 2004.

18. Wu LW, Mayo LD, Dunbar JD, et al: Utilization of distinct signaling pathways by receptors for vascular endothelial cell growth factor and other mitogens in the induction of endothelial cell proliferation. J Biol Chem 275: 5096-5103, 2000.
19. Hamano Y, Zeisberg M, Sugimoto H, et al: Physiological levels of tumstatin, a fragment of collagen IV alpha3 chain, are generated by MMP-9 proteolysis and suppress angiogenesis via alphaV beta3 integrin. Cancer Cell 3: 589-601, 2003.

20. Mayoral R, Fernandez-Martinez A, Bosca L and Martin-Sanz P. Prostaglandin E2 promotes migration and adhesion in hepatocellular carcinoma cells. Carcinogenesis 26: 753-761, 2005.

21. Nejjari M, Hafdi Z, Gouysse G, et al: Expression, regulation, and function of alpha $\mathrm{V}$ integrins in hepatocellular carcinoma: an in vivo and in vitro study. Hepatology 36: 418-426, 2002.

22. Pasco S, Ramont L, Venteo L, Pluot M, Maquart FX and Monboisse JC: In vivo overexpression of tumstatin domains by tumor cells inhibits their invasive properties in a mouse melanoma model. Exp Cell Res 301: 251-265, 2004.

23. Maeshima Y, Sudhakar A, Lively JC, et al: Tumstatin, an endothelial cell-specific inhibitor of protein synthesis. Science 295: 140-143, 2002.

24. Sudhakar A, Sugimoto H, Yang C, Lively J, Zeisberg M and Kalluri R: Human tumstatin and human endostatin exhibit distinct antiangiogenic activities mediated by alpha $\mathrm{v}$ beta 3 and alpha 5 beta 1 integrins. Proc Natl Acad Sci USA 100: 4766-4771, 2003.

25. Kalluri R: Basement membranes: structure, assembly and role in tumour angiogenesis. Nat Rev Cancer 3: 422-433, 2003.

26. Bagli E, Stefaniotou M, Morbidelli L, et al: Luteolin inhibits vascular endothelial growth factor-induced angiogenesis; inhibition of endothelial cell survival and proliferation by targeting phosphatidylinositol 3'-kinase activity. Cancer Res 64: 7936-7946, 2004.

27. Hood JD, Frausto R, Kiosses WB, Schwartz MA and Cheresh DA Differential alphav integrin-mediated Ras-ERK signaling during two pathways of angiogenesis. J Cell Biol 162: 933-943, 2003. 